\title{
Existence of Solutions for Functional Integral Equation Involving the Henstock-Kurzweil-Stieltjes Integral
}

\author{
Hui $\mathrm{Mei}^{1}$, Guoju Ye ${ }^{1}$, Wei Liu ${ }^{1} \&$ Yanrong Chen ${ }^{1}$ \\ ${ }^{1}$ College of Science, Hohai University, P. R. China \\ Correspondence: Hui Mei, College of Science, Hohai University, Nanjing 210098, P. R. China. \\ E-mail: meihuihhu@163.com
}

Received: July 24, 2017 Accepted: August 14, 2017 Online Published: September 5, 2017

doi:10.5539/jmr.v9n5p46 URL: https://doi.org/10.5539/jmr.v9n5p46

\begin{abstract}
In this paper, we apply the method associated with the technique of measure of noncompactness and some generalizations of Darbo fixed points theorem to study the existence of solutions for a class of integral equation involving the HenstockKurzweil-Stieltjes integral. Meanwhile, an example is provided to illustrate our results.
\end{abstract}

Keywords: Henstock-Kurzweil-Stieltjes integral, measure of noncompactness, Darbo fixed point theorem, coupled fixed point

2010 MSC: 47H08, 26A39.

\section{Introduction}

Existence theorems of coupled fixed points have been considered by several authors (Chang \& Cho, 1996; Roshan, 2017). In (Chang \& Cho, 1996), the authors proved the existence of coupled fixed points for a class of integral operator:

$$
A(u, v)(t)=h(t, u(t), v(t))+\int_{0}^{t} K(t, s) \psi(s, u(s), v(s)) d s,
$$

where $t \in[0, L], L>0, u, v \in C[0, L], K \in C([0, L] \times[0, L]), h, \psi \in C\left([0, L] \times \mathbb{R}_{+} \times \mathbb{R}_{+}\right), \mathbb{R}_{+}=[0, \infty), C[0, L]$ is the space of all real valued continuous functions on $[0, L]$.

In this paper we establish the existence of solutions for the following integral equation involving the Henstock-KurzweilStieltjes integral:

$$
(x, y)(t)=h(t, x(t), y(t))+\phi\left(t, \int_{0}^{t} f(t, s, x(s), y(s)) d g(s)\right),
$$

where $h, \phi, f$ are continuous functions, $g:[0, L] \rightarrow \mathbb{R}$ is of boundary variation. $d g$ can be identified with a Stieltjes measure and will have the effect of suddenly changing the state of the system at the points of discontinuity of $g$, that is, the system could be controlled by some impulsive force. The Henstock-Kurzweil-Stieltjes integral, which is a generalization of the Lebesgue-Stieltjes integral (Krejčí, 2006; Kurzweil, 1957; Lee, 1989; Schwabik \& Ye, 2005), has been proved useful in the study of ordinary differential equations (Chew, 1988; Chew \& Flordeliza, 1991; Heikkilä \& Ye, 2012; Ye \& Liu, 2016).

To achieve our goal, the approach associated with the technique of measure of noncompactness and some generalizations of Darbo fixed points theorem will be used.

This paper is organized as follows. In Section 2, we recall some basic concepts of the Henstock-Kurzweil-Stieltjes integrals and measure of noncompactness. In Section 3, we verify the existence of solutions for (2) by a coupled fixed point theorem. In Section 4, we give an example to illustrate Theorem 3.2 in this paper.

\section{Preliminaries}

In this section, so that the paper is self-contained, we provide preliminary material with respect to the Henstock-KurzweilStieltjes integral and measure of noncompactness.

\subsection{The Henstock-Kurzweil-Stieltjes Integral}

The basic concept in the Henstock-Kurzweil-Stieltjes integration theory is that of a $\delta$-fine partition, we refer the interested reader to (Krejčí, 2006; Kurzweil, 1957; Lee, 1989; Schwabik \& Ye, 2005). 
Now, we introduce the definition of Henstock-Kurzweil-Stieltjes integrals.

For given functions $f, g:[a, b] \rightarrow \mathbb{R}$ and a $\delta$-fine partition $D$, we define

$$
\begin{aligned}
K_{D}(f, g) & =\sum_{i=1}^{m} f\left(\xi_{i}\right)\left(g\left(t_{i}\right)-g\left(t_{i-1}\right)\right) \\
& =f(b) g(b)-f(a) g(a)-\sum_{i=1}^{m}\left(f\left(\xi_{i}\right)-f\left(\xi_{i-1}\right)\right) g\left(t_{i-1}\right),
\end{aligned}
$$

where $\xi_{0}=a, \xi_{m}=b$.

Definition 2.1. (Krejčí, 2006) Let $f, g:[a, b] \rightarrow \mathbb{R}$ be given. We say that $J \in \mathbb{R}$ is the Henstock-Kurzweil-Stieltjes integral $(H K S)$ over $[a, b]$ of $f$ with respect to $g$ and denote

$$
J=(H K S) \int_{a}^{b} f(t) d g(t)=(H K S) \int_{a}^{b} f d g
$$

if for every $\varepsilon>0$, there exists positive function $\delta>0$, such that for every $\delta$-fine $D$, we have

$$
\left|J-K_{D}(f, g)\right| \leq \varepsilon .
$$

Definition 2.2. (Krejčí, 2006) A function $f:[a, b] \rightarrow \mathbb{R}$ is called regulated on $[a, b]$, if the limits

$$
\lim _{s \rightarrow t-} f(s)=f(t-), t \in(a, b], \text { and } \lim _{s \rightarrow t+} f(s)=f(t+), t \in[a, b)
$$

exist and are finite with the convention

$$
f(a-)=f(a), \quad f(b+)=f(b) .
$$

Denote by $G[a, b]$ the space of all real valued regulated functions on $[a, b]$.

Obviously, both the space $C[a, b]$ of all real valued continuous functions on $[a, b]$ and the space $B V[a, b]$ of all functions of bounded variation on $[a, b]$ are subsets of $G[a, b]$.

Lemma 2.3. (Krejčí, 2006) If $f \in G[a, b], g \in B V[a, b]$, then both $\int_{a}^{b} f d g$ and $\int_{a}^{b} g d f$ exist, and

$$
\begin{aligned}
& \left|\int_{a}^{b} f(t) d g(t)\right| \leq\|f\| \cdot \operatorname{Var}_{[a, b]} g, \\
& \left|\int_{a}^{b} g(t) d f(t)\right| \leq\|f\|\left(|g(a)|+|g(b)|+\operatorname{Var}_{[a, b]} g\right) .
\end{aligned}
$$

Moreover, if $f_{n} \in G[a, b], g_{n} \in B V[a, b]$ are such that $\lim _{n \rightarrow \infty}\left\|f_{n}-f\right\|=0, \lim _{n \rightarrow \infty}\left\|g_{n}-g\right\|=0$ as $n \rightarrow \infty$, and $\underset{[a, b]}{\operatorname{Var}} g_{n} \leq C$ independently of $n$, then

$$
\lim _{n \rightarrow \infty} \int_{a}^{b} f_{n}(t) d g_{n}(t)=\int_{a}^{b} f(t) d g(t)
$$

\subsection{Measure of Noncompactness}

In this subsection, we recall some fundamental facts concerning measure of noncompactness (see[Banaś \& Goebel(1980)]). Let $(E,\|\cdot\|)$ be a real Banach space with zero element 0 and $B(x, r)$ denote the closed ball in $E$ centered at $x$ with radius $r$. The symbol $B_{r}$ stands for the ball $B(0, r)$. Denote by $\bar{X}, \overline{c o n v} X$ the closure and the closed convex hull of a nonempty subset $X$ of $E$ singly. Finally, let us denote by $\mathfrak{m}_{E}$ the family of all nonempty and bounded subsets of $E$ and by $\mathfrak{n}_{E}$ its subfamilies consisting of all relatively compact subsets.

Definition 2.4. (Mursaleen, 2017) Let $(E, d)$ be a metric space and $X$ a bounded subset of $E$. The Hausdorff measure of noncompactness ( $\mu$-measure or ball measure of noncompactness) of the set $X$, denoted by $\mu(X)$ is defined to be the infimum of the set of all reals $\varepsilon>0$ such that $X$ can be covered by a finite number of balls of radii $<\varepsilon$, that is,

$$
\mu(X)=\inf \{\varepsilon>0: X \text { has a finite } \varepsilon \text {-net in } E\} .
$$

The function $\mu$ is called the Hausdorff measure of noncompactness. 
For each $x \in C[0, L]$, we define

$$
\omega(x, \varepsilon)=\sup \{|x(t)-x(s)|: t, s \in[0, L],|t-s| \leq \varepsilon\} .
$$

Obviously, $\omega(x, \varepsilon) \rightarrow 0$, as $\varepsilon \rightarrow 0$, since $x$ is uniformly continuous on $[0, L]$. Moreover, if this limit relation holds uniformly for $x$ running over some bounded set $X \subset C[0, L]$, then $X$ is equicontinuous, and vice versa. Therefore, we have:

Theorem 2.5. On the space $C[0, L]$, the measure of noncompactness (3) is equivalent to

$$
\mu(X)=\lim _{\varepsilon \rightarrow 0} \sup _{x \in X} \omega(x, \varepsilon)
$$

for all bounded sets $X \subset C[0, L]$.

Remark 2.6. For $X \subset C([0, L] \times[0, L]), \mu(X)$ can be defined similarly, see (Kazemi \& Ezzati, 2016, Theorem 2.2).

Definition 2.7. (Chang \& Cho, 1996) An element $(x, y) \in X \times X$ is called a coupled fixed point of a mapping $T: X \times X \rightarrow X$ if $T(x, y)=x$ and $T(y, x)=y$.

Lemma 2.8. (Banaś \& Goebel, 1980, Theorem 2) Let $\Omega$ be a nonempty, bounded, closed and convex subset of a Banach space $E$ and let $T: \Omega \rightarrow \Omega$ be a continuous mapping. Assume that there exists a constant $k \in[0,1)$ such that

$$
\mu(T(X)) \leq k \mu(X)
$$

for any $X \subset \Omega$. Then $T$ has a fixed point.

Denote by $\Phi$ the class of all continuous functions $\varphi: \mathbb{R}_{+} \times \mathbb{R}_{+} \rightarrow \mathbb{R}_{+}$satisfying

(1) for all $u_{1}, v_{1}, u_{2}, v_{2} \in \mathbb{R}_{+}, \varphi\left(u_{1}, v_{1}\right) \leq \varphi\left(u_{2}, v_{2}\right)$ if $u_{1} \leq u_{2}, v_{1} \leq v_{2}$;

(2) $\varphi(u, u)<u$ for all $u>0$;

(3) $\frac{1}{2} \varphi\left(u_{1}, v_{1}\right)+\frac{1}{2} \varphi\left(u_{2}, v_{2}\right) \leq \varphi\left(\frac{u_{1}+u_{2}}{2}, \frac{v_{1}+v_{2}}{2}\right)$ for all $u_{1}, v_{1}, u_{2}, v_{2} \in \mathbb{R}_{+}$.

See details in (Roshan, 2017).

The following generalization of Darbo fixed point theorem will be needed in Section 3.

Lemma 2.9. (Roshan, 2017, Theorem 3.7) Let $\Omega$ be a nonempty, bounded, closed and convex subset of a Banach space $E$, $\mu$ be an arbitrary measure of noncompactness. Moreover assume that $T: \Omega \times \Omega \rightarrow \Omega$ be a continuous function satisfying

$$
\mu\left(T\left(X_{1} \times X_{2}\right)\right) \leq \varphi\left(\mu\left(X_{1}\right), \mu\left(X_{2}\right)\right)
$$

for all $X_{1}, X_{2} \subseteq \Omega \times \Omega$, where $\varphi \in \Phi$. Then $T$ has at least a coupled fixed point.

\section{Main Results}

In this section, we shall prove the existence of solutions of Eq. (2).

Firstly, we give the following assumptions:

$\left(D_{1}\right)$ The function $h:[0, L] \times \mathbb{R} \times \mathbb{R} \rightarrow \mathbb{R}$ is continuous and there exists a function $\varphi \in \Phi$ such that

$$
|h(t, x, y)-h(t, u, v)| \leq \varphi(|x-u|,|y-v|), \forall t \in[0, L], \forall x, y, u, v \in \mathbb{R},
$$

and $M_{1}=\sup \{|h(t, 0,0)|: t \in[0, L]\} ;$

$\left(D_{2}\right)$ The function $\phi:[0, L] \times \mathbb{R} \rightarrow \mathbb{R}$ is continuous,

$$
\left|\phi\left(t, z_{1}\right)-\phi\left(t, z_{2}\right)\right| \leq\left|z_{1}-z_{2}\right|, \forall t \in[0, L], \forall z_{1}, z_{2} \in \mathbb{R},
$$

and $M_{2}=\sup \{|\phi(t, 0)|: t \in[0, L]\}$ 
$\left(D_{3}\right)$ The function $g \in B V[0, L] \cap C[0, L]$ is nondecreasing, the function $f:[0, L] \times[0, L] \times \mathbb{R} \times \mathbb{R} \rightarrow \mathbb{R}$ is continuous, and there exist functions $m_{1}:[0, L] \rightarrow \mathbb{R}_{+}$and $m_{2}:[0, L] \rightarrow \mathbb{R}_{+}$are continuous, such that

$$
|f(t, s, x, y)| \leq m_{1}(t) m_{2}(s),
$$

and $M_{3}=\sup \left\{m_{1}(t) \int_{0}^{t} m_{2}(s) d g(s)\right\}$, for any $t, s \in[0, L]$ such that $s \leq t$, and for each $x \in \mathbb{R}$;

$\left(D_{4}\right)$ There exists $r>0$ such that

$$
M_{1}+M_{2}+M_{3}+\varphi(r, r) \leq r .
$$

Let $C[0, L] \times C[0, L]$ be equipped with the norm $\|(x, y)\|=\|x\|+\|y\|$. We define an operator $F$ on $C[0, L] \times C[0, L]$ by

$$
F(x, y)(t)=h(t, x(t), y(t))+\phi\left(t, \int_{0}^{t} f(t, s, x(s), y(s)) d g(s)\right) .
$$

Then we have the following statement.

Theorem 3.1. Under the assumptions $\left(D_{1}\right)-\left(D_{4}\right)$, the operator $F$ given in $(5)$ has at least one coupled fixed point in the space $C[0, L] \times C[0, L]$.

Proof. (i) For any $(x, y) \in C[0, L] \times C[0, L]$, and $\|x\|,\|y\| \leq r$,

$$
\begin{aligned}
|F(x, y)(t)| \leq & |h(t, x(t), y(t))|+\left|\phi\left(t, \int_{0}^{t} f(t, s, x(s), y(s)) d g(s)\right)\right| \\
\leq & |h(t, x(t), y(t))-h(t, 0,0)|+|h(t, 0,0)| \\
& +\left|\phi\left(t, \int_{0}^{t} f(t, s, x(s), y(s)) d g(s)\right)-\phi(t, 0)\right|+|\phi(t, 0)| \\
\leq & \varphi(|x|,|y|)+M_{1}+\left|\int_{0}^{t} f(t, s, x(s), y(s)) d g(s)\right|+M_{2} \\
\leq & M_{1}+M_{2}+m_{1}(t) \int_{0}^{t} m_{2}(s) d g(s)+\varphi(\|x\|,\|y\|) \\
\leq & M_{1}+M_{2}+M_{3}+\varphi(\|x\|,\|y\|) \\
\leq & r .
\end{aligned}
$$

This implies that $F$ maps the space $B_{r} \times B_{r}$ into $B_{r}$, where $B_{r}=\{x, y \in C[0, L]:\|x\| \leq r,\|y\| \leq r\}, r$ is a constant arising in assumption $\left(D_{4}\right)$.

(ii) We prove that the operator $F$ is continuous on $B_{r} \times B_{r}$.

For arbitrary $(x, y) \in B_{r} \times B_{r}, \varepsilon>0$, now let $(u, v) \in B_{r} \times B_{r}$ with $\|(x, y)-(u, v)\|<\varepsilon$, then we have

$$
\begin{aligned}
& |F(x, y)(t)-F(u, v)(t)| \\
\leq & |h(t, x(t), y(t))-h(t, u(t), v(t))| \\
& +\left|\phi\left(t, \int_{0}^{t} f(t, s, x(s), y(s)) d g(s)\right)-\phi\left(t, \int_{0}^{t} f(t, s, u(s), v(s)) d g(s)\right)\right| \\
\leq & \varphi(|x-u|,|y-v|)+\left|\int_{0}^{t} f(t, s, x(s), y(s)) d g(s)-\int_{0}^{t} f(t, s, u(s), v(s)) d g(s)\right| \\
\leq & \varphi(\|x-u\|,\|y-v\|)+\int_{0}^{t}|f(t, s, x(s), y(s))-f(t, s, u(s), v(s))| d g(s) \\
\leq & \varphi(\varepsilon, \varepsilon)+\int_{0}^{L} \omega_{1}(f, \varepsilon) d g(s) \\
\leq & \varphi(\varepsilon, \varepsilon)++\left\|\omega_{1}(f, \varepsilon)\right\| \cdot \operatorname{Var}_{[0, L]} g,
\end{aligned}
$$

where

$$
\omega_{1}(f, \varepsilon)=\sup \{|f(t, s, x(s), y(s))-f(t, s, u(s), v(s))|, t, s \in[0, L],\|(x, y)-(u, v)\| \leq \varepsilon\} .
$$

Since uniformly continuity of the function $t \mapsto f(t, s, x, y)$ on the set $[0, L]$, we infer that $\omega_{1}(f, \varepsilon) \rightarrow 0$ as $\varepsilon \rightarrow 0$. 
Thus, taking into account the property of the function $\varphi$ and linking (6), for each $t \in[0, L]$ we get

$$
|F(x, y)(t)-F(u, v)(t)| \leq \varepsilon .
$$

Hence, the operator $F$ is continuous on $B_{r} \times B_{r}$.

(iii) Taking arbitrary nonempty subsets $X_{1}, X_{2}$ of the ball $B_{r}$. Fix $\varepsilon>0$, choose arbitrarily $t_{1}, t_{2} \in[0, L]$ such that $\left|t_{1}-t_{2}\right| \leq \varepsilon$. Without loss of generality, assuming that $t_{2}<t_{1}$. Then, for arbitrary $(x, y) \in X_{1} \times X_{2}$, we get

$$
\begin{aligned}
& \left|F(x, y)\left(t_{1}\right)-F(x, y)\left(t_{2}\right)\right| \\
\leq & \left|h\left(t_{1}, x\left(t_{1}\right), y\left(t_{1}\right)\right)-h\left(t_{2}, x\left(t_{2}\right), y\left(t_{2}\right)\right)\right| \\
& +\left|\phi\left(t_{1}, \int_{0}^{t_{1}} f\left(t_{1}, s, x(s), y(s)\right) d g(s)\right)-\phi\left(t_{2}, \int_{0}^{t_{2}} f\left(t_{2}, s, x(s), y(s)\right) d g(s)\right)\right| \\
\leq & \left|h\left(t_{1}, x\left(t_{1}\right), y\left(t_{1}\right)\right)-h\left(t_{1}, x\left(t_{2}\right), y\left(t_{2}\right)\right)\right|+\left|h\left(t_{1}, x\left(t_{2}\right), y\left(t_{2}\right)\right)-h\left(t_{2}, x\left(t_{2}\right), y\left(t_{2}\right)\right)\right| \\
& +\left|\phi\left(t_{1}, \int_{0}^{t_{1}} f\left(t_{1}, s, x(s), y(s)\right) d g(s)\right)-\phi\left(t_{1}, \int_{0}^{t_{1}} f\left(t_{2}, s, x(s), y(s)\right) d g(s)\right)\right| \\
& +\left|\phi\left(t_{1}, \int_{0}^{t_{1}} f\left(t_{2}, s, x(s), y(s)\right) d g(s)\right)-\phi\left(t_{2}, \int_{0}^{t_{1}} f\left(t_{2}, s, x(s), y(s)\right) d g(s)\right)\right| \\
& +\left|\phi\left(t_{2}, \int_{0}^{t_{1}} f\left(t_{2}, s, x(s), y(s)\right) d g(s)\right)-\phi\left(t_{2}, \int_{0}^{t_{2}} f\left(t_{2}, s, x(s), y(s)\right) d g(s)\right)\right| \\
\leq & \varphi\left(\left|x\left(t_{1}\right)-x\left(t_{2}\right)\right|,\left|y\left(t_{1}\right)-y\left(t_{2}\right)\right|\right)+\omega(h, \varepsilon) \\
& +\left|\int_{0}^{t_{1}} f\left(t_{1}, s, x(s), y(s)\right) d g(s)-\int_{0}^{t_{1}} f\left(t_{2}, s, x(s), y(s)\right) d g(s)\right|+\omega(\phi, \varepsilon) \\
& +\left|\int_{0}^{t_{1}} f\left(t_{2}, s, x(s), y(s)\right) d g(s)-\int_{0}^{t_{2}} f\left(t_{2}, s, x(s), y(s)\right) d g(s)\right| \\
\leq & \varphi(\omega(x, \varepsilon), \omega(y, \varepsilon))+\omega(h, \varepsilon)+\int_{0}^{t_{1}} \omega(f, \varepsilon) d g(s)+\omega(\phi, \varepsilon) \\
& +\int_{t_{2}}^{t_{1}}\left|f\left(t_{2}, s, x(s), y(s)\right)\right| d g(s) \\
\leq & \varphi(\omega(x, \varepsilon), \omega(y, \varepsilon))+\omega(h, \varepsilon)+\int_{0}^{t_{1}} \omega(f, \varepsilon) d g(s)+\omega(\phi, \varepsilon) \\
+ & m_{1}(t) \int_{t_{2}}^{t_{1}} m_{2}(s) d g(s),
\end{aligned}
$$

where

$$
\begin{aligned}
& \omega(h, \varepsilon)=\sup \left\{\left|h\left(t_{1}, x, y\right)-h\left(t_{2}, x, y\right)\right|: t_{1}, t_{2} \in[0, L],\left|t_{1}-t_{2}\right| \leq \varepsilon, x, y \in[-r, r]\right\}, \\
& \omega(\phi, \varepsilon)=\sup \left\{\left|\phi\left(t_{1}, z\right)-\phi\left(t_{2}, z\right)\right|: t_{1}, t_{2} \in[0, L],\left|t_{1}-t_{2}\right| \leq \varepsilon\right\}, \\
& \omega(f, \varepsilon)=\sup \left\{\left|f\left(t_{1}, s, x, y\right)-f\left(t_{2}, s, x, y\right)\right|: t_{1}, t_{2} \in[0, L],\left|t_{1}-t_{2}\right| \leq \varepsilon, x, y \in[-r, r]\right\}, \\
& \omega(x, \varepsilon)=\sup \left\{\left|x\left(t_{1}\right)-x\left(t_{2}\right)\right|: t_{1}, t_{2} \in[0, L],\left|t_{1}-t_{2}\right| \leq \varepsilon\right\}, \\
& \omega(y, \varepsilon)=\sup \left\{\left|y\left(t_{1}\right)-y\left(t_{2}\right)\right|: t_{1}, t_{2} \in[0, L],\left|t_{1}-t_{2}\right| \leq \varepsilon\right\},
\end{aligned}
$$

By $\left(D_{1}\right)-\left(D_{3}\right), h, f, \phi$ are uniformly continuous on $[0, L]$, so

$$
\omega(h, \varepsilon) \rightarrow 0, \omega(f, \varepsilon) \rightarrow 0, \omega(\phi, \varepsilon) \rightarrow 0 \text { as } \varepsilon \rightarrow 0 .
$$

Moreover, since the functions $m_{1}(t), m_{2}(s)$ are continuous, we have

$$
m_{1}(t) \int_{t_{2}}^{t_{1}} m_{2}(s) d g(s) \rightarrow 0 \text { as }\left|t_{1}-t_{2}\right| \rightarrow 0 .
$$

Since $(x, y)$ is an arbitrary element of $X_{1} \times X_{2}$ in (8), we obtain

$$
\omega\left(F\left(X_{1} \times X_{2}\right), \varepsilon\right) \leq \varphi(\omega(x, \varepsilon), \omega(y, \varepsilon)) .
$$


Hence,

$$
\lim _{\varepsilon \rightarrow 0} \sup \omega\left(F\left(X_{1} \times X_{2}\right), \varepsilon\right) \leq \varphi\left(\lim _{\varepsilon \rightarrow 0} \sup \omega(x, \varepsilon), \lim _{\varepsilon \rightarrow 0} \sup \omega(y, \varepsilon)\right) .
$$

It follows from (9) and Theorem 2.5 that

$$
\mu\left(F\left(X_{1} \times X_{2}\right)\right) \leq \varphi\left(\mu\left(X_{1}\right), \mu\left(X_{2}\right)\right)
$$

According to Lemma 2.9, $F$ has at least a coupled fixed point in the space $B_{r} \times B_{r}$. The proof is therefore complete.

According to Theorem 3.1 and (5) the definition of the operator $F$, we have

Theorem 3.2. Under the assumptions $\left(D_{1}\right)-\left(D_{4}\right)$, Eq. (2) has at least one solution in the space $C[0, L] \times C[0, L]$.

\section{Applications}

Example 4.1. Consider the following integral equation

$$
\begin{aligned}
(x, y)(t)= & \frac{1}{8} e^{-t}+\frac{1}{2} \ln (1+|x(t)|)+\frac{1}{2} \ln (1+|y(t)|)+\frac{t^{2}}{2\left(1+t^{2}\right)} \\
& +2 \ln \left(1+\frac{\left|\int_{0}^{t} e^{-t^{2}} \cdot \frac{\cos x(s) \cos y(s)}{(1+|\sin x(s)|)(1+|\sin y(s)|)} d g(s)\right|}{2}\right), t \in[0,1],
\end{aligned}
$$

where $g$ is the Cantor-Lebesgue function (Dovgoshey, Martio, Ryazanov \& Vuorinen, 2006).

It is obvious that Eq. (11) is a exception of Eq. (2) with

$$
\begin{aligned}
h(t, x, y) & =\frac{1}{8} e^{-t}+\frac{1}{2} \ln (1+|x(t)|)+\frac{1}{2} \ln (1+|y(t)|), \\
\phi(t, z) & =\frac{t^{2}}{2\left(1+t^{2}\right)}+2 \ln \left(1+\frac{|z|}{2}\right), \\
f(t, s, x, y) & =e^{-t^{2}} \cdot \frac{\cos x(s) \cos y(s)}{(1+|\sin x(s)|)(1+|\sin y(s)|)}, \\
\varphi(t, s) & =\ln \left(1+\frac{t+s}{2}\right) \in \Phi .
\end{aligned}
$$

Now we show that all the conditions of Theorem 3.2 are satisfied for Eq. (11).

(i) Obviously, $h$ and $\phi$ are continuous.

(ii) Clearly, the function $|h(t, 0,0)|=\frac{1}{8} e^{-t}$, so $M_{1}=\frac{1}{8}$.

(iii) The function $|\phi(t, 0)|=\frac{t^{2}}{2\left(1+t^{2}\right)}$, so $M_{2}=\frac{1}{2}$.

(iv) Suppose that $t \in[0,1]$ and $x, y, u, v, z_{1}, z_{2} \in \mathbb{R}$ with $|x| \geq|u|,|y| \geq|v|$. Then we can get

$$
\begin{aligned}
|h(t, x, y)-h(t, u, v)| & =\frac{1}{2}(\ln (1+|x|)-\ln (1+|u|))+\frac{1}{2}(\ln (1+|y|)-\ln (1+|v|)) \\
& =\frac{1}{2} \ln \left(\frac{1+|x|}{1+|u|}\right)+\frac{1}{2} \ln \left(\frac{1+|y|}{1+|v|}\right) \\
& =\frac{1}{2} \ln \left(1+\frac{|x|-|u|}{1+|u|}\right)+\frac{1}{2} \ln \left(1+\frac{|y|-|v|}{1+|v|}\right) \\
& \leq \frac{1}{2} \ln (1+|x-u|)+\frac{1}{2} \ln (1+|y-v|) \\
& \leq \ln \left(1+\frac{|x-u|+|y-v|}{2}\right) \\
& =\varphi(|x-u|,|y-v|) .
\end{aligned}
$$


Moreover we can get

$$
\begin{aligned}
\left|\phi\left(t, z_{1}\right)-\phi\left(t, z_{2}\right)\right| & =2\left|\left(\ln \left(1+\frac{\left|z_{1}\right|}{2}\right)-\ln \left(1+\frac{\left|z_{2}\right|}{2}\right)\right)\right| \\
& \leq\left|z_{1}-z_{2}\right| .
\end{aligned}
$$

(v) Further, notice that the function $f$ is continuous, and we have

$$
|f(t, s, x, y)| \leq e^{-t^{2}}
$$

for $t, s \in[0,1]$ and $x, y \in \mathbb{R}$. If we put $m_{1}(t)=e^{-t^{2}}, m_{2}(s)=1$, then we have $M_{3}=\sup \left\{e^{-t^{2}} \int_{0}^{t} d g(s): t \in[0,1]\right\}<1$.

(vi) It is easy to check that for each number $r \geq 5$, we have the following inequality

$$
M_{1}+M_{2}+M_{3}+\varphi(r, r)<\frac{1}{8}+\frac{1}{2}+1+\ln (1+r)<r .
$$

Consequently, all the conditions of Theorem 3.2 are satisfied and Eq. (11) has at least one solution in the space $C[0,1] \times$ $C[0,1]$.

Remark 4.2. In Example 4.1, the Cantor-Lebesgue function $g \in C[0,1] \cap B V[0,1]$, but $g$ is not absolutely continuous on $[0,1]$. Therefore, the methods used to deal with integral equations involving the Lebesgue (or Riemann) integral (Chang \& Cho, 1996; Roshan, 2017) are no longer applicable in this case. This means our existence result Theorem 3.2 is more general.

\section{Conclusions}

In this research, by using the approach associated with the technique of measure of noncompactness and some generalizations of Darbo fixed points theorem, we studied the existence of solutions for a class of integral equation involving the Henstock-Kurzweil-Stieltjes integral, and we obtained the existence of at least one solution for the functional integral equation we considered.

\section{Acknowledgements}

This work is supported by the Fundamental Research Funds for the Central Universities.

\section{References}

Banaś, J., \& Goebel, K. (1980). Measures of noncompactness in Banach spaces. Marcel Dekker, New York.

Chang, S. S., Cho, Y. J., \& Huang, N. J. (1996). Coupled fixed point theorems with applications. J. Korean Math. Soc., $33,575-585$.

Chew, T. S. (1988). On Kurzweil generalized ordinary differential equations. J. Differential Equations, 76, $286-293$. http://doi.org/10.1016/0022-0396(88)90076-9

Chew, T. S., \& Flordeliza, F. (1991). On $x^{\prime}=f(t, x)$ and Henstock-Kurzweil integrals. Differential Integral Equations, 4 , 861-868.

Dovgoshey, O., Martio, O., Ryazanov, V., \& Vuorinen, M. (2006). The Cantor function. Expo. Math., 24 , 1-37. http://doi.org/10.1016/j.exmath.2005.05.002

Heikkilä, S., \& Ye, G. (2012). Equations containing locally Henstock-Kurzweil integrable functions. Appl. Math., 57, 569-580. http://doi.org/10.1007/s10492-012-0034-7

Kazemi, M., \& Ezzati, R. (2016). Existence of solution for some nonlinear two-dimensional Volterra integral equations via measures of noncompactness. Appl. Math. Comput., 275, 165-171. http://doi.org/10.1016/j.amc.2015.11.066

Krejčí, P. (2006). The Kurzweil integral and hysteresis. J. Phys.: Conf. Ser., 55, 144-154.

Kurzweil, J. (1957). Generalized ordinary differential equations and continuous dependence on a parameter. Czechoslovak Math. J., 7, 418-449.

Lee, P. Y. (1989). Lanzhou lectures on Henstock integration. World Scientific, Singapore.

Mursaleen, M. (2017). Differential equations in classical sequence spaces. Rev. R. Acad. Cienc. Exactas Fís. Nat. Ser. A Math. RACSAM, 111, 587-612. http://doi.org/10.1007/s13398-016-0301-7 
Roshan, J. R. (2017). Existence of solutions for a class of system of functional integral equation via measure of noncompactness. J. Comput. Appl. Math., 313, 129-141. http://doi.org/10.1016/j.cam.2016.09.011

Schwabik, Š., \& Ye, G. (2005). Topics in Banach space integration. World Scientific, Singapore. http://dx.doi.org/10.1142/9789812703286

Ye, G., \& Liu, W. (2016). The distributional Henstock-Kurzweil integral and applications. Monatsh. Math., 181, 975-989. http://doi.org/10.4208/jms.v49n4.16.06

\section{Copyrights}

Copyright for this article is retained by the author(s), with first publication rights granted to the journal.

This is an open-access article distributed under the terms and conditions of the Creative Commons Attribution license (http://creativecommons.org/licenses/by/4.0/). 